\title{
A NEW SYSTEM OF GENERALIZED NONLINEAR RELAXED COCOERCIVE VARIATIONAL INEQUALITIES
}

\author{
KE DING, WEN-YONG YAN, AND NAN-JING HUANG
}

Received 21 November 2004; Revised 13 April 2005; Accepted 28 June 2005

We introduce and study a new system of generalized nonlinear relaxed cocoercive inequality problems and construct an iterative algorithm for approximating the solutions of the system of generalized relaxed cocoercive variational inequalities in Hilbert spaces. We prove the existence of the solutions for the system of generalized relaxed cocoercive variational inequality problems and the convergence of iterative sequences generated by the algorithm. We also study the convergence and stability of a new perturbed iterative algorithm for approximating the solution.

Copyright (c) 2006 Ke Ding et al. This is an open access article distributed under the Creative Commons Attribution License, which permits unrestricted use, distribution, and reproduction in any medium, provided the original work is properly cited.

\section{Introduction}

Variational inequality problems have various applications in mechanics and physics, optimization and control, linear and nonlinear programming, economics and finance, transportation equilibrium and engineering science, and so forth. Consequently considerable attention has been devoted to the study of the theory and efficient numerical methods for variational inequality problems (see, e.g., [2-17] and the references therein). In [15], Verma introduced a new system of nonlinear strongly monotone variational inequalities and studied the approximate of this system based on the projection method, and in [16], Verma discussed the approximate solvability of a system of nonlinear relaxed cocoercive variational inequalities in Hilbert spaces. Recently, Kim and Kim [14] introduced and studied a system of nonlinear mixed variational inequalities in Hilbert spaces, and obtained some approximate solvability results. In the recent paper [6], Cho et al. introduced and studied a new system of nonlinear variational inequalities in Hilbert spaces. They proved some existence and uniqueness theorems of solutions for the system of nonlinear variational inequalities. They also constructed an iterative algorithm for approximating the solution of the system of nonlinear variational inequalities. Some related works, we refer to $[2,3,5,7-10,12,13]$. Motivated and inspired by these works, in this paper, we introduce and study a new system of generalized nonlinear relaxed cocoercive variational 
inequality problems and construct an iterative algorithm for approximating the solutions of the system of generalized relaxed cocoercive variational inequalities in Hilbert spaces. We prove the existence of the solutions for the system of generalized relaxed cocoercive variational inequality problems and the convergence of iterative sequences generated by the algorithm. We also study the convergence and stability of a new perturbed iterative algorithm for approximating the solution. The results presented in this paper improve and extend the previously known results in this area.

\section{Preliminaries}

Let $H$ be a Hilbert space endowed with a norm $\|\cdot\|$ and inner product $(\cdot, \cdot)$, respectively. Let $\mathrm{CB}(H)$ be the family of all nonempty subsets of $H$ and $K_{1}, K_{2}$ be two convex and closed subsets of $H$. Let $g_{1}, g_{2}, m_{1}, m_{2}: H \rightarrow H$ and $F, G: H \times H \rightarrow H$ be mappings. We consider the following system of generalized nonlinear variational inequality problems: find $x, y \in H$ such that $g_{i}(x) \in K_{i}(x)$ for $i=1,2$, and

$$
\begin{aligned}
& \left(F(x, y), z-g_{1}(x)\right) \geq 0, \quad \forall z \in K_{1}(x), \\
& \left(G(x, y), z-g_{2}(y)\right) \geq 0, \quad \forall z \in K_{2}(y),
\end{aligned}
$$

where $K_{i}(x)=m_{i}(x)+K_{i}$ for $i=1,2$.

When $K_{1}$ and $K_{2}$ are both convex cones of $H$, it is easy to see that problem (2.1) is equivalent to the following system of generalized nonlinear co-complementarity problems: find $x, y \in H$ such that $g_{i}(x) \in K_{i}(x)$ for $i=1,2$, and

$$
\begin{gathered}
F(x, y) \in\left(K_{1}(x)-g_{1}(x)\right)^{*}, \\
G(x, y) \in\left(K_{2}(y)-g_{2}(y)\right)^{*},
\end{gathered}
$$

where $K_{i}(x)=m_{i}(x)+K_{i}$ and $\left(K_{i}(x)-g_{i}(x)\right) *$ is the dual of $K_{i}(x)-g_{i}(x)$ for $i=1,2$, that is,

$$
\left(K_{i}(x)-g_{i}(x)\right)^{*}=\left\{u \in H \mid(u, v) \geq 0, \forall v \in K_{i}(x)-g_{i}(x)\right\}
$$

Some examples of problems (2.1) and (2.2) are as follows.

(I) If $G=0$ and $F(x, y)=T x+A x$ for all $x, y \in X$, where $T, A: H \rightarrow H$ are two mappings, then problem (2.2) reduces to finding $x \in H$ such that

$$
T x+A x \in\left(K_{1}(x)-g_{1}(x)\right)^{*},
$$

which is called the generalized complementarity problem. The problem (2.4) was extended and studied by Jou and Yao [11] in Hilbert spaces, and by Chen et al. [5] in the setting of Banach spaces.

(II) Let $T: H \times H \rightarrow H$ be a mapping. If $F(x, y)=\rho T(y, x)+x-y, G(x, y)=\eta T(x, y)$ $+y-x$ for all $x, y \in H, m_{1}=m_{2}=0, K_{1}=K_{2}=K$, and $g_{1}=g_{2}=I$, where $I$ is an identity 
Ke Ding et al. 3

mapping and $\rho>0, \eta>0$, then problem (2.1) reduces to finding $x, y \in K$ such that

$$
\begin{aligned}
& (\rho T(y, x)+x-y, z-x) \geq 0, \quad \forall z \in K, \\
& (\eta T(x, y)+y-x, z-y) \geq 0, \quad \forall z \in K,
\end{aligned}
$$

which is called the system of nonlinear variational inequality problems considered by Verma [16]. The special case of problem (2.5) was studied by Verma [15]. The problem (2.5) was extended and studied by Agarwal et al. [1], Kim and Kim [14], and Cho et al. [6].

(III) If $m_{1}=m_{2}=0$, and $g_{1}=g_{2}=I$, then problem (2.1) reduces to finding $x \in K_{1}$ and $y \in K_{2}$ such that

$$
\begin{aligned}
& (F(x, y), z-x) \geq 0, \quad \forall z \in K_{1}, \\
& (G(x, y), z-y) \geq 0, \quad \forall z \in K_{2},
\end{aligned}
$$

which is just the problem considered in [12] with $F, G$ being single-valued mappings.

Definition 2.1. A mapping $N: H \times H \rightarrow H$ is said to be

(i) $\alpha$-strongly monotone with respect to first argument if there exists some $\alpha>0$ such that

$$
(N(x, \cdot)-N(y, \cdot), x-y) \geq \alpha\|x-y\|^{2}, \quad \forall(x, y) \in H \times H
$$

(ii) $\xi$-Lipschitz continuous with respect to the first argument, if there exists a constant $\xi>0$ such that

$$
\|N(x, \cdot)-N(y, \cdot)\| \leq \xi\|x-y\|, \quad \forall(x, y) \in H \times H .
$$

Similarly, we can define the strong monotonicity and Lipschitzian continuity with respect to the second argument of $N$.

Definition 2.2. A Mapping $N: H \times H \rightarrow H$ is said to be relaxed $(a, b)$-cocoercive with respect to the first argument if there exists constants $a>0$ and $b>0$ such that

$$
(N(x, \cdot)-N(y, \cdot), x-y) \geq(-a)\|x-y\|^{2}+b\|x-y\|^{2}, \quad \forall(x, y) \in H \times H .
$$

If $a=0$, then $N$ is $b$-strongly monotone. Similarly, we can define the relaxed $(a, b)$-cocoercivity with respect to the second argument of $N$.

LEMma 2.3 [4]. If $K \subset H$ is a closed convex subset and $z \in H$ is a given point, then there exists $x \in K$ such that

$$
(x-z, y-x) \geq 0, \quad \forall y \in K
$$

if and only if $x=P_{K} z$, where $P_{K}$ is the projection of $H$ onto $K$.

Lemma 2.4 [4]. The projection $P_{K}$ is nonexpansive, that is,

$$
\left\|P_{K} u-P_{K} v\right\| \leq\|u-v\|, \quad \forall u, v \in H .
$$


4 Nonlinear relaxed cocoercive variational inequalities

Lemma 2.5 [18]. Let $\left\{K_{n}\right\}$ be a sequence of closed convex subsets of $H$ such that $H\left(K_{n}, K\right) \rightarrow$ 0 as $n \rightarrow \infty$, where $H(\cdot, \cdot)$ is the Hausdorff metric, that is, for any $A, B \in \mathrm{CB}(H)$,

$$
H(A, B)=\max \left\{\sup _{a \in A} \inf _{b \in B}\|a-b\|, \sup _{b \in B} \inf _{a \in A}\|a-b\|\right\} .
$$

Then

$$
\left\|P_{K_{n}} v-P_{K} v\right\| \longrightarrow 0 \quad(n \longrightarrow \infty), \forall v \in H .
$$

Lemma 2.6 [4]. If $K(u)=m(u)+K$ for all $u \in H$, then

$$
P_{K(u)} v=m(u)+P_{K}(v-m(u)) .
$$

From Lemmas 2.3 and 2.6, we have the following lemma.

Lemma 2.7. If $K_{1}, K_{2} \subset H$ are two closed convex cones, and $K_{i}(\cdot)=m(\cdot)+K_{i}(i=1,2)$, then $x, y \in H$ solve problem (2.1) if and only if $x, y \in H$ such that

$$
\begin{aligned}
& x=x-g_{1}(x)+m_{1}(x)+P_{K_{1}}\left(g_{1}(x)-\rho F(x, y)-m_{1}(x)\right), \\
& y=y-g_{2}(y)+m_{2}(y)+P_{K_{2}}\left(g_{2}(y)-\rho G(x, y)-m_{2}(y)\right),
\end{aligned}
$$

where $\rho>0$ is a constant.

Lемма 2.8 [17]. Let $\left\{\mu_{n}\right\}$ be a real sequence of nonnegative numbers and $\left\{\nu_{n}\right\}$ be a real sequence of numbers in $[0,1]$ with $\sum_{n=0}^{\infty} v_{n}=\infty$. If there exists a constant $n_{1}$ such that

$$
\mu_{n+1} \leq\left(1-v_{n}\right) \mu_{n}+v_{n} \delta_{n}, \quad \forall n \geq n_{1},
$$

where $\delta_{n} \geq 0$ for all $n \geq 0$, and $\delta_{n} \rightarrow 0(n \rightarrow \infty)$, then $\lim _{n \rightarrow \infty} \mu_{n}=0$.

\section{Existence and convergence}

In this section, we construct an iterative algorithm to approximate the solution of problem (2.1) and study the convergence of the sequence generated by the algorithm.

Algorithm 3.1. For any given $x_{0}, y_{0} \in H$, we compute

$$
\begin{aligned}
& x_{n+1}=x_{n}-g_{1}\left(x_{n}\right)+m_{1}\left(x_{n}\right)+P_{K_{1}}\left(g_{1}\left(x_{n}\right)-\rho F\left(x_{n}, y_{n}\right)-m_{1}\left(x_{n}\right)\right), \\
& y_{n+1}=y_{n}-g_{2}\left(y_{n}\right)+m_{2}\left(y_{n}\right)+P_{K_{2}}\left(g_{2}\left(y_{n}\right)-\rho G\left(x_{n}, y_{n}\right)-m_{2}\left(y_{n}\right)\right) .
\end{aligned}
$$

Theorem 3.2. Let $g_{i}: H \rightarrow H$ be $\eta_{i}$-strongly monotone and $\zeta_{i}$-Lipschitz continuous and $m_{i}: H \rightarrow H$ be $\gamma_{i}$-Lipschitz continuous $(i=1,2)$. Let $F: H \times H \rightarrow H$ be $l_{1}, l_{2}$-Lipschitz continuous with respect to the first, second arguments, respectively, and relaxed $(a, b)$-cocoercive with respect to the first argument. Let $G: H \times H \rightarrow H$ be $n_{1}, n_{2}$-Lipschitz continuous with respect to the first, second arguments, respectively, and relaxed $(c, d)$-cocoercive with respect 
Ke Ding et al. 5

to the second argument. If

$$
\begin{aligned}
& 2 \sqrt{1+\zeta_{1}^{2}-2 \eta_{1}}+2 \gamma_{1}+\sqrt{1+\rho^{2} l_{1}^{2}+2 \rho a l_{1}^{2}-2 \rho b}+\rho n_{1}<1, \\
& 2 \sqrt{1+\zeta_{2}^{2}-2 \eta_{2}}+2 \gamma_{2}+\sqrt{1+\rho^{2} n_{2}^{2}+2 \rho c n_{2}^{2}-2 \rho d}+\rho l_{2}<1 .
\end{aligned}
$$

then there exist $x^{*}, y^{*} \in H$, which solve problem (2.1). Moreover, the iterative sequences $\left\{x_{n}\right\}$ and $\left\{y_{n}\right\}$ generated by Algorithm 3.1 converge to $x^{*}$ and $y^{*}$, respectively.

Proof. From (3.1) and Lemma 2.6, we have

$$
\begin{gathered}
\left\|x_{n+1}-x_{n}\right\|=\| x_{n}-g_{1}\left(x_{n}\right)+m_{1}\left(x_{n}\right)+P_{K_{1}}\left(g_{1}\left(x_{n}\right)-\rho F\left(x_{n}, y_{n}\right)-m_{1}\left(x_{n}\right)\right) \\
-\left[x_{n-1}-g_{1}\left(x_{n-1}\right)+m_{1}\left(x_{n-1}\right)\right. \\
\left.\quad+P_{K_{1}}\left(g_{1}\left(x_{n-1}\right)-\rho F\left(x_{n-1}, y_{n-1}\right)-m_{1}\left(x_{n-1}\right)\right)\right] \| \\
\leq\left\|x_{n}-x_{n-1}-\left(g_{1}\left(x_{n}\right)-g_{1}\left(x_{n-1}\right)\right)\right\|+\left\|m_{1}\left(x_{n}\right)-m_{1}\left(x_{n-1}\right)\right\| \\
+\| P_{K_{1}}\left(g_{1}\left(x_{n}\right)-\rho F\left(x_{n}, y_{n}\right)-m_{1}\left(x_{n}\right)\right) \\
\quad-P_{K_{1}}\left(g_{1}\left(x_{n-1}\right)-\rho F\left(x_{n-1}, y_{n-1}\right)-m_{1}\left(x_{n-1}\right)\right) \| .
\end{gathered}
$$

Since $g_{1}$ is $\zeta_{1}$-Lipschitz continuous and $\eta_{1}$-strongly monotone,

$$
\left\|x_{n}-x_{n-1}-\left(g_{1}\left(x_{n}\right)-g_{1}\left(x_{n-1}\right)\right)\right\|^{2} \leq\left(1+\zeta_{1}^{2}-2 \eta_{1}\right)\left\|x_{n}-x_{n-1}\right\|^{2} .
$$

From the $\gamma_{1}$-Lipschitzian continuity of $m_{1}$, we have

$$
\left\|m_{1}\left(x_{n}\right)-m_{1}\left(x_{n-1}\right)\right\| \leq \gamma_{1}\left\|x_{n}-x_{n-1}\right\| \text {. }
$$

Lemma 2.4 implies that $P_{K_{1}}$ is nonexpansive and it follows from the strong monotonicity of $g_{1}$ that

$$
\begin{aligned}
\| P_{K_{1}} & \left(g_{1}\left(x_{n}\right)-\rho F\left(x_{n}, y_{n}\right)-m_{1}\left(x_{n}\right)\right)-P_{K_{1}}\left(g_{1}\left(x_{n-1}\right)-\rho F\left(x_{n-1}, y_{n-1}\right)-m_{1}\left(x_{n-1}\right)\right) \| \\
\leq & \left\|\left(g_{1}\left(x_{n}\right)-\rho F\left(x_{n}, y_{n}\right)-m_{1}\left(x_{n}\right)\right)-\left(g_{1}\left(x_{n-1}\right)-\rho F\left(x_{n-1}, y_{n-1}\right)-m_{1}\left(x_{n-1}\right)\right)\right\| \\
\leq & \left\|x_{n}-x_{n-1}-\left(g_{1}\left(x_{n}\right)-g_{1}\left(x_{n-1}\right)\right)\right\|+\left\|m_{1}\left(x_{n}\right)-m_{1}\left(x_{n-1}\right)\right\| \\
& \quad+\left\|x_{n}-x_{n-1}-\rho\left(F\left(x_{n}, y_{n}\right)-F\left(x_{n-1}, y_{n}\right)\right)\right\|+\rho\left\|F\left(x_{n-1}, y_{n}\right)-F\left(x_{n-1}, y_{n-1}\right)\right\| .
\end{aligned}
$$


6 Nonlinear relaxed cocoercive variational inequalities

Since $F$ is relaxed $(a, b)$-cocoercive and $l_{1}$-Lipschitz continuous with respect to the first argument,

$$
\begin{aligned}
\| x_{n}- & x_{n-1}-\rho\left(F\left(x_{n}, y_{n}\right)-F\left(x_{n-1}, y_{n}\right)\right) \|^{2} \\
= & \left\|x_{n}-x_{n-1}\right\|^{2}+\rho^{2}\left\|F\left(x_{n}, y_{n}\right)-F\left(x_{n-1}, y_{n}\right)\right\|^{2} \\
& \quad-2\left(x_{n}-x_{n-1}, \rho\left(F\left(x_{n}, y_{n}\right)-F\left(x_{n-1}, y_{n}\right)\right)\right) \\
\leq & \left\|x_{n}-x_{n-1}\right\|^{2}+\rho^{2}\left\|F\left(x_{n}, y_{n}\right)-F\left(x_{n-1}, y_{n}\right)\right\|^{2} \\
& +2 \rho a\left\|F\left(x_{n}, y_{n}\right)-F\left(x_{n-1}, y_{n}\right)\right\|^{2}-2 \rho b\left\|x_{n}-x_{n-1}\right\|^{2} \\
= & \left(1+l_{1}^{2} \rho^{2}+2 \rho a l_{1}^{2}-2 \rho b\right)\left\|x_{n}-x_{n-1}\right\|^{2} .
\end{aligned}
$$

Since $F$ is $l_{2}$-Lipschitz continuous with respect to the second argument,

$$
\left\|F\left(x_{n-1}, y_{n}\right)-F\left(x_{n-1}, y_{n-1}\right)\right\| \leq l_{2}\left\|y_{n}-y_{n-1}\right\| .
$$

It follows from (3.3)-(3.8) that

$$
\begin{aligned}
& \left\|x_{n+1}-x_{n}\right\| \\
& \quad \leq\left(2 \sqrt{1+\zeta_{1}^{2}-2 \eta_{1}}+2 \gamma_{1}+\sqrt{1+\rho^{2} l_{1}^{2}+2 \rho a l_{1}^{2}-2 \rho b}\right)\left\|x_{n}-x_{n-1}\right\|+\rho l_{2}\left\|y_{n}-y_{n-1}\right\| .
\end{aligned}
$$

Similarly, we have

$$
\begin{aligned}
& \left\|y_{n+1}-y_{n}\right\| \\
& \quad \leq\left(2 \sqrt{1+\zeta_{2}^{2}-2 \eta_{2}}+2 \gamma_{2}+\sqrt{1+\rho^{2} n_{2}^{2}+2 \rho c n_{2}^{2}-2 \rho d}\right)\left\|y_{n}-y_{n-1}\right\|+\rho n_{1}\left\|x_{n}-x_{n-1}\right\| .
\end{aligned}
$$

Now (3.9) and (3.10) imply

$$
\begin{aligned}
& \left\|x_{n+1}-x_{n}\right\|+\left\|y_{n+1}-y_{n}\right\| \\
& \leq\left(2 \sqrt{1+\zeta_{1}^{2}-2 \eta_{1}}+2 \gamma_{1}+\sqrt{1+\rho^{2} l_{1}^{2}+2 \rho a l_{1}^{2}-2 \rho b}+\rho n_{1}\right)\left\|x_{n}-x_{n-1}\right\| \\
& +\left(2 \sqrt{1+\zeta_{2}^{2}-2 \eta_{2}}+2 \gamma_{2}+\sqrt{1+\rho^{2} n_{2}^{2}+2 \rho c n_{2}^{2}-2 \rho d}+\rho l_{2}\right), \\
& \left\|y_{n}-y_{n-1}\right\| \leq \omega\left(\left\|x_{n}-x_{n-1}\right\|+\left\|y_{n}-y_{n-1}\right\|\right),
\end{aligned}
$$

where

$$
\begin{aligned}
\omega=\max \left\{2 \sqrt{1+\zeta_{1}^{2}-2 \eta_{1}}+2 \gamma_{1}+\sqrt{1+\rho^{2} l_{1}^{2}+2 \rho a l_{1}^{2}-2 \rho b}+\rho n_{1},\right. \\
\left.2 \sqrt{1+\zeta_{2}^{2}-2 \eta_{2}}+2 \gamma_{2}+\sqrt{1+\rho^{2} n_{2}^{2}+2 \rho c n_{2}^{2}-2 \rho d}+\rho l_{2}\right\} .
\end{aligned}
$$


It follows from (3.2) that $\omega<1$. Thus (3.11) implies that $\left\{x_{n}\right\}$ and $\left\{y_{n}\right\}$ are both Cauchy sequences in $H$, and $\left\{x_{n}\right\}$ converges to $x^{*} \in H,\left\{y_{n}\right\}$ converges to $y^{*} \in H$. Since $m_{1}, m_{2}$, $g_{1}, g_{2}, P_{K_{1}}, P_{K_{2}}, F, G$ are all continuous, we have

$$
\begin{aligned}
& x^{*}=x^{*}-g_{1}\left(x^{*}\right)+m_{1}\left(x^{*}\right)+P_{K}\left(g_{1}\left(x^{*}\right)-\rho F\left(x^{*}, y^{*}\right)-m_{1}\left(x^{*}\right)\right), \\
& y^{*}=y^{*}-g_{2}\left(y^{*}\right)+m_{2}\left(y^{*}\right)+P_{K}\left(g_{2}\left(y^{*}\right)-\rho G\left(x^{*}, y^{*}\right)-m_{2}(y *)\right),
\end{aligned}
$$

The result follows then from Lemma 2.7. This completes the proof.

Remark 3.3. Let $\rho>0$ be a number satisfying the conditions.

$$
\begin{aligned}
\mid \rho- & \frac{b-a l_{1}^{2}-\left(1-e_{1}\right) n_{1}}{l_{1}^{2}-n_{1}^{2}} \mid \\
& <\frac{\left(1-e_{1}\right)^{2}-1+\left(\left(b-a l_{1}^{2}-\left(1-e_{1}\right) n_{1}\right)^{2}\right) /\left(l_{1}^{2}-n_{1}^{2}\right)}{l_{1}^{2}-n_{1}^{2}}, \quad \rho n_{1}<1-e_{1}, n_{1}<l_{1}, \\
\mid \rho- & \frac{d-c n_{2}^{2}-\left(1-e_{2}\right) l_{2}}{n_{2}^{2}-l_{2}^{2}} \mid \\
& <\frac{\left(1-e_{2}\right)^{2}-1+\left(\left(d-c n_{2}^{2}-\left(1-e_{2}\right) l_{2}\right)^{2}\right) /\left(n_{2}^{2}-l_{2}^{2}\right)}{n_{2}^{2}-l_{2}^{2}}, \quad \rho l_{2}<1-e_{2}, l_{2}<n_{2},
\end{aligned}
$$

where $e_{1}=2 \sqrt{1+\zeta_{1}^{2}-2 \eta_{1}}+2 \gamma_{1}$ and $e_{2}=2 \sqrt{1+\zeta_{2}^{2}-2 \eta_{2}}+2 \gamma_{2}$. Then (3.2) holds.

\section{Perturbed algorithm and stability}

In this section, we construct a new perturbed iterative algorithm for solving problem (2.1) and prove the convergence and stability of the iterative sequence generated by the algorithm.

Definition 4.1. Let $T$ be a self-map of $H, x_{0} \in H$ and let $x_{n+1}=f\left(T, x_{n}\right)$ define an iteration procedure which yields a sequence of points $\left\{x_{n}\right\}_{n=0}^{\infty}$ in $H$. Suppose that $\{x \in H: T x=$ $x\} \neq \varnothing$ and $\left\{x_{n}\right\}_{n=0}^{\infty}$ converge to a fixed point $x^{*}$ of $T$. Let $\left\{u_{n}\right\} \subset H$ and let $\epsilon_{n}=\| u_{n+1}-$ $f\left(T, u_{n}\right) \|$. If $\lim \epsilon_{n}=0$ implies that $\lim u_{n}=x^{*}$, then the iteration procedure defined by $x_{n+1}=f\left(T, x_{n}\right)$ is said to be $T$-stable or stable with respect to $T$. Some results for the stability of various iterative processes, we refer to $[1,10]$ and the references therein.

Let $\left\{K_{n}^{1}\right\}$ and $\left\{K_{n}^{2}\right\}$ be two sequences of closed convex subsets of $H$ such that $H\left(K_{n}^{1}, K\right) \rightarrow 0, H\left(K_{n}^{2}, K\right) \rightarrow 0$, when $n \rightarrow \infty$. Now we consider the following perturbed algorithm for solving problem (2.1). 
8 Nonlinear relaxed cocoercive variational inequalities

Algorithm 4.2. For any given $x_{0}, y_{0} \in H$, we compute

$$
\begin{gathered}
x_{n+1}=\left(1-t_{n}\right) x_{n}+t_{n}\left(x_{n}-g_{1}\left(x_{n}\right)+m_{1}\left(x_{n}\right)+P_{K_{n}^{1}}\left(g_{1}\left(x_{n}\right)-\rho F\left(x_{n}, y_{n}\right)-m_{1}\left(x_{n}\right)\right)\right)+t_{n} e_{n}, \\
y_{n+1}=\left(1-t_{n}\right) y_{n}+t_{n}\left(y_{n}-g_{2}\left(y_{n}\right)+m_{2}\left(y_{n}\right)+P_{K_{n}^{2}}\left(g_{2}\left(y_{n}\right)-\rho G\left(x_{n}, y_{n}\right)-m_{2}\left(y_{n}\right)\right)\right)+t_{n} j_{n},
\end{gathered}
$$

for all $n=0,1,2, \ldots$, where $\left\{e_{n}\right\}$ and $\left\{j_{n}\right\}$ are two sequences of the elements of $H$, and the sequence $\left\{t_{n}\right\}$ satisfies the following conditions

$$
0 \leq t_{n} \leq 1, \quad \forall n \geq 0, \quad \sum_{n=0}^{\infty} t_{n}=\infty .
$$

Let $\left\{u_{n}\right\}$ and $\left\{v_{n}\right\}$ be any sequences in $H$ and define $\epsilon_{n}=\epsilon_{n}^{1}+\epsilon_{n}^{2}$ by

$$
\begin{aligned}
\epsilon_{n}^{1}=\| u_{n+1}-\left\{\left(1-t_{n}\right) u_{n}+t_{n}[\right. & u_{n}-g_{1}\left(u_{n}\right)+m_{1}\left(u_{n}\right) \\
& \left.\left.+P_{K_{1}}\left(g_{1}\left(u_{n}\right)-\rho F\left(u_{n}, v_{n}\right)+m_{1}\left(u_{n}\right)\right)\right]+t_{n} e_{n}\right\} \| \\
\epsilon_{n}^{2}=\| v_{n+1}-\left\{\left(1-t_{n}\right) v_{n}+t_{n}[\right. & v_{n}-g_{2}\left(v_{n}\right)+m_{2}\left(v_{n}\right) \\
+ & \left.\left.P_{K_{2}}\left(g_{2}\left(v_{n}\right)-\rho G\left(u_{n}, v_{n}\right)+m_{2}\left(v_{n}\right)\right)\right]+t_{n} j_{n}\right\} \| .
\end{aligned}
$$

Theorem 4.3. Let $g_{i}: X \rightarrow X$ be $\eta_{i}$-strongly monotone and $\zeta_{i}$-Lipschitz continuous, and $m_{i}: X \rightarrow X$ be $\tau_{i}$-Lipschitz continuous for $i=1,2$. Let $F: X \times X \rightarrow X$ be $l_{1}, l_{2}$-Lipschitz continuous with respect to the first and second arguments, respectively, and relaxed $(a, b)$ cocoercive with respect to the first argument. Let $G: X \times X \rightarrow X$ be $n_{1}, n_{2}$-Lipschitz continuous with respect to the first and second arguments, respectively, and relaxed $(c, d)$-cocoercive with respect to the second argument. Suppose $H\left(K_{n}, K\right) \rightarrow 0(n \rightarrow \infty)$ and

$$
\begin{aligned}
& \left|\rho-\frac{b-a l_{1}^{2}-\left(1-e_{1}\right) n_{1}}{l_{1}^{2}-n_{1}^{2}}\right| \\
& \quad<\frac{\left(1-e_{1}\right)^{2}-1+\left(\left(b-a l_{1}^{2}-\left(1-e_{1}\right) n_{1}\right)^{2}\right) /\left(l_{1}^{2}-n_{1}^{2}\right)}{l_{1}^{2}-n_{1}^{2}}, \quad \rho n_{1}<1-e_{1}, n_{1}<l_{1}, \\
& \left|\rho-\frac{d-c n_{2}^{2}-\left(1-e_{2}\right) l_{2}}{n_{2}^{2}-l_{2}^{2}}\right| \\
& \quad<\frac{\left(1-e_{2}\right)^{2}-1+\left(\left(d-c n_{2}^{2}-\left(1-e_{2}\right) l_{2}\right)^{2}\right) /\left(n_{2}^{2}-l_{2}^{2}\right)}{n_{2}^{2}-l_{2}^{2}}, \quad \rho l_{2}<1-e_{2}, l_{2}<n_{2},
\end{aligned}
$$

where $e_{i}=2 \sqrt{1+\zeta_{i}^{2}-2 \eta_{i}}+2 \gamma_{i}$ for $i=1$, 2 . If $\lim _{n \rightarrow \infty}\left\|e_{n}\right\|=0$ and $\lim _{n \rightarrow \infty}\left\|j_{n}\right\|=0$, then we have the following conclusions.

(I) The iterative sequences generated by Algorithm 4.2 converge to the unique solution of (2.1).

(II) Moreover, if $0<t \leq t_{n}$, then $\lim u_{n}=x^{*}, \lim v_{n}=y^{*}$ if and only if $\lim \left(\epsilon_{n}^{1}+\epsilon_{n}^{2}\right)=0$, where $\epsilon_{n}^{1}$ and $\epsilon_{n}^{2}$ are defined by (4.3). 
Ke Ding et al. 9

Proof. By Theorem 3.2, problem (2.1) admits a solution $\left(x^{*}, y^{*}\right)$. It is easy to prove that $\left(x^{*}, y^{*}\right)$ is the unique solution of (4.1). From Lemma 2.7, we have

$$
\begin{aligned}
& x^{*}=\left(1-t_{n}\right) x^{*}+t_{n}\left(x^{*}-g_{1}\left(x^{*}\right)+m_{1}\left(x^{*}\right)+P_{K_{1}}\left(g_{1}\left(x^{*}\right)-\rho F\left(x^{*}, y^{*}\right)-m_{1}\left(x^{*}\right)\right)\right), \\
& y^{*}=\left(1-t_{n}\right) y^{*}+t_{n}\left(y^{*}-g_{2}\left(y^{*}\right)+m_{2}\left(y^{*}\right)+P_{K_{2}}\left(g_{2}\left(y^{*}\right)-\rho G\left(x^{*}, y^{*}\right)-m_{2}(y *)\right)\right),
\end{aligned}
$$

Since $P_{K}$ is nonexpansive and it follows from (4.1) and (4.5) that

$$
\begin{aligned}
\| x_{n+1} & -x^{*} \| \\
= & \|\left(1-t_{n}\right) x_{n}+t_{n}\left[x_{n}-g_{1}\left(x_{n}\right)+m_{1}\left(x_{n}\right)+P_{K_{n}^{1}}\left(g_{1}\left(x_{n}\right)-\rho F\left(x_{n}, y_{n}\right)-m_{1}\left(x_{n}\right)\right)\right]+t_{n} e_{n} \\
& -\left(1-t_{n}\right) x^{*}-t_{n}\left[x^{*}-g_{1}\left(x^{*}\right)+m_{1}\left(x^{*}\right)+P_{K_{1}}\left(g_{1}\left(x^{*}\right)-\rho F\left(x^{*}, y^{*}\right)-m_{1}\left(x^{*}\right)\right)\right] \| \\
\leq & \left(1-t_{n}\right)\left\|\left(x_{n}-x^{*}\right)\right\|+t_{n}\left\|\left(x_{n}-x^{*}\right)+g_{1}\left(x_{n}\right)-g_{1}\left(x^{*}\right)\right\|+t_{n}\left\|m_{1}(x)-m_{1}\left(x^{*}\right)\right\|+t_{n}\left\|e_{n}\right\| \\
& +t_{n}\left\|P_{K_{n}^{1}}\left(g_{1}\left(x_{n}\right)-\rho F\left(x_{n}, y_{n}\right)-m_{1}\left(x_{n}\right)\right)-P_{K_{1}}\left(g_{1}\left(x^{*}\right)-\rho F\left(x^{*}, y^{*}\right)-m_{1}\left(x^{*}\right)\right)\right\| \\
\leq & \left(1-t_{n}\right)\left\|\left(x_{n}-x^{*}\right)\right\|+t_{n}\left\|\left(x_{n}-x^{*}\right)+g_{1}\left(x_{n}\right)-g_{1}\left(x^{*}\right)\right\|+t_{n}\left\|m_{1}(x)-m_{1}\left(x^{*}\right)\right\|+t_{n}\left\|e_{n}\right\| \\
& +t_{n}\left\|P_{K_{n}^{1}}\left(g_{1}\left(x_{n}\right)-\rho F\left(x_{n}, y_{n}\right)-m_{1}\left(x_{n}\right)\right)-P_{K_{n}^{1}}\left(g_{1}\left(x^{*}\right)-\rho F\left(x^{*}, y^{*}\right)-m_{1}\left(x^{*}\right)\right)\right\| \\
& +t_{n}\left\|P_{K_{n}^{1}}\left(g_{1}\left(x^{*}\right)-\rho F\left(x^{*}, y^{*}\right)-m_{1}\left(x^{*}\right)\right)-P_{K_{1}}\left(g_{1}\left(x^{*}\right)-\rho F\left(x^{*}, y^{*}\right)-m_{1}\left(x^{*}\right)\right)\right\| \\
\leq & \left(1-t_{n}\right)\left\|\left(x_{n}-x^{*}\right)\right\|+t_{n}\left\|\left(x_{n}-x^{*}\right)+g_{1}\left(x_{n}\right)-g_{1}\left(x^{*}\right)\right\|+t_{n}\left\|m_{1}(x)-m_{1}\left(x^{*}\right)\right\|+t_{n}\left\|e_{n}\right\| \\
& +t_{n}\left\|x_{n}-x^{*}-\left(g_{1}\left(x_{n}\right)-g_{1}\left(x^{*}\right)\right)\right\|+t_{n}\left\|m_{1}\left(x_{n}\right)-m_{1}\left(x^{*}\right)\right\| \\
& +t_{n}\left\|x_{n}-x^{*}-\rho\left(F\left(x_{n}, y_{n}\right)-F\left(x^{*}, y_{n}\right)\right)\right\|+\rho t_{n}\left\|F\left(x^{*}, y_{n}\right)-F\left(x^{*}, y^{*}\right)\right\| \\
& +t_{n}\left\|P_{K_{n}^{1}}\left(g_{1}\left(x^{*}\right)-\rho F\left(x^{*}, y^{*}\right)-m_{1}\left(x^{*}\right)\right)-P_{K_{1}}\left(g_{1}\left(x^{*}\right)-\rho F\left(x^{*}, y^{*}\right)-m_{1}\left(x^{*}\right)\right)\right\| .
\end{aligned}
$$

Since $F$ is $l_{2}$-Lipschitz continuous with respect to the second argument,

$$
\left\|F\left(x^{*}, y_{n}\right)-F\left(x^{*}, y^{*}\right)\right\| \leq l_{2}\left\|y_{n}-y^{*}\right\| .
$$

From the strong monotonicity and Lipschitzian continuity of $g_{1}$, we obtain

$$
\left\|x_{n}-x^{*}-\left(g_{1}\left(x_{n}\right)-g_{1}\left(x^{*}\right)\right)\right\|^{2} \leq\left(1+\zeta_{1}^{2}-2 \eta_{1}\right)\left\|x_{n}-x^{*}\right\|^{2} .
$$

The Lipschitzian continuity of $m_{1}$ implies

$$
\left\|m_{1}\left(x_{n}\right)-m_{1}\left(x^{*}\right)\right\| \leq \gamma_{1}\left\|x_{n}-x^{*}\right\| .
$$


10 Nonlinear relaxed cocoercive variational inequalities

Since $F$ is relaxed $(a, b)$-cocoercive and $l_{1}$-Lipschitz continuous with respect to the first argument,

$$
\left\|x_{n}-x^{*}-\rho\left(F\left(x_{n}, y_{n}\right)-F\left(x^{*}, y_{n}\right)\right)\right\| \leq \sqrt{1+\rho^{2} l_{1}^{2}+2 \rho a l_{1}^{2}-2 \rho b}\left\|x_{n}-x^{*}\right\| .
$$

It follows from (4.6)-(4.10) that

$$
\begin{aligned}
\left\|x_{n+1}-x^{*}\right\| \leq & \left(2 t_{n} \sqrt{1+\zeta_{1}^{2}-2 \eta_{1}}+2 t_{n} \gamma_{1}+t_{n} \sqrt{1+\rho^{2} l_{1}^{2}+2 \rho a l_{1}^{2}-2 \rho b}+1-t_{n}\right)\left\|x_{n}-x^{*}\right\| \\
& +t_{n} \rho l_{2}\left\|y_{n}-y^{*}\right\|+t_{n} b_{n}+t_{n}\left\|e_{n}\right\|,
\end{aligned}
$$

where

$$
b_{n}=\left\|P_{K_{n}^{1}}\left(g_{1}\left(x^{*}\right)-\rho F\left(x^{*}, y^{*}\right)-m_{1}\left(x^{*}\right)\right)-P_{K_{1}}\left(g_{1}\left(x^{*}\right)-\rho F\left(x^{*}, y^{*}\right)-m_{1}\left(x^{*}\right)\right)\right\| \text {. }
$$

From the fact of $H\left(K_{n}^{1}, K_{1}\right) \rightarrow 0$ and Lemma 2.5, we know that $b_{n} \rightarrow 0$.

Similarly, we have

$$
\begin{aligned}
\left\|y_{n+1}-y^{*}\right\| \leq & \left(2 t_{n} \sqrt{1+\zeta_{2}^{2}-2 \eta_{2}}+2 t_{n} \gamma_{2}+t_{n} \sqrt{1+\rho^{2} n_{2}^{2}+2 \rho c n_{2}^{2}-2 \rho d}+1-t_{n}\right)\left\|y_{n}-y^{*}\right\| \\
& +t_{n} \rho n_{1}\left\|x_{n}-x^{*}\right\|+t_{n} c_{n}+t_{n}\left\|j_{n}\right\|,
\end{aligned}
$$

where

$$
c_{n}=\left\|P_{K_{n}^{2}}\left(g_{2}\left(y^{*}\right)-\rho G\left(x^{*}, y^{*}\right)-m_{2}\left(y^{*}\right)\right)-P_{K_{2}}\left(g_{2}\left(y^{*}\right)-\rho F\left(x^{*}, y^{*}\right)-m_{2}\left(y^{*}\right)\right)\right\| \text {, }
$$

and $c_{n} \rightarrow 0$. Now (4.11) and (4.13) imply

$$
\begin{aligned}
\| x_{n+1}- & x^{*}\|+\| y_{n+1}-y^{*} \| \\
\leq & \left(2 t_{n} \sqrt{1+\zeta_{1}^{2}-2 \eta_{1}}+2 t_{n} \gamma_{1}+1-t_{n}+t_{n} \sqrt{1+\rho^{2} l_{1}^{2}+2 \rho a l_{1}^{2}-2 \rho b}+t_{n} \rho n_{1}\right)\left\|x_{n}-x^{*}\right\| \\
& +\left(2 t_{n} \sqrt{1+\zeta_{2}^{2}-2 \eta_{2}}+2 t_{n} \gamma_{2}+1-t_{n}+t_{n} \sqrt{1+\rho^{2} n_{2}^{2}+2 \rho c n_{2}^{2}-2 \rho d}+t_{n} \rho l_{2}\right)\left\|y_{n}-y^{*}\right\| \\
& +t_{n} c_{n}+t_{n} b_{n}+t_{n}\left\|e_{n}\right\|+t_{n}\left\|j_{n}\right\| .
\end{aligned}
$$


Ke Ding et al. 11

Let

$$
\begin{aligned}
& h_{1}=2 \sqrt{1+\zeta_{1}^{2}-2 \eta_{1}}+2 \gamma_{1}+\sqrt{1+\rho^{2} l_{1}^{2}+2 \rho a l_{1}^{2}-2 \rho b}+\rho n_{1}, \\
& h_{2}=2 \sqrt{1+\zeta_{2}^{2}-2 \eta_{2}}+2 \gamma_{2}+\sqrt{1+\rho^{2} n_{2}^{2}+2 \rho c n_{2}^{2}-2 \rho d}+\rho l_{3} .
\end{aligned}
$$

From (4.4), it is easy to see that $0 \leq h_{1}<1$ and $0 \leq h_{2}<1$. Let $h=\max \left\{h_{1}, h_{2}\right\}$. Then $h<1$ and so (4.15) reduces to

$$
\begin{aligned}
\| x_{n+1} & -x^{*}\|+\| y_{n+1}-y^{*} \| \\
& \leq\left(1-(1-h) t_{n}\right)\left(\left\|x_{n}-x^{*}\right\|+\left\|y_{n}-y^{*}\right\|\right)+t_{n}\left(b_{n}+c_{n}+\left\|e_{n}\right\|+\left\|j_{n}\right\|\right) \\
& =\left(1-(1-h) t_{n}\right)\left(\left\|x_{n}-x^{*}\right\|+\left\|y_{n}-y^{*}\right\|\right)+(1-h) t_{n} \delta_{n},
\end{aligned}
$$

where

$$
\delta_{n}=\frac{b_{n}+c_{n}+\left\|e_{n}\right\|+\left\|j_{n}\right\|}{1-h}
$$

From (4.12), (4.14) and Lemma 2.5, we have

$$
b_{n} \longrightarrow 0, \quad c_{n} \longrightarrow 0, \quad \delta_{n}=\frac{b_{n}+c_{n}+\left\|e_{n}\right\|+\left\|j_{n}\right\|}{1-h} \longrightarrow 0 \quad(n \longrightarrow \infty)
$$

It follows from (4.2), (4.17), (4.19) and Lemma 2.8 that

$$
x_{n} \longrightarrow x^{*}, \quad y_{n} \longrightarrow y^{*} \quad(n \longrightarrow \infty)
$$

This completes the proof of Conclusion I.

Next we prove Conclusion II. By using (4.1), we obtain

$$
\begin{aligned}
& \left\|u_{n+1}-x^{*}\right\| \\
& \leq \quad\left\|u_{n+1}-\left\{\left(1-t_{n}\right) u_{n}+t_{n}\left[u_{n}-g_{1}\left(u_{n}\right)+m_{1}\left(u_{n}\right)+P_{K_{1}}\left(g_{1}\left(u_{n}\right)-\rho F\left(u_{n}, v_{n}\right)+m_{1}\left(u_{n}\right)\right)\right]+t_{n} e_{n}\right\}\right\| \\
& \quad+\left\|\left\{\left(1-t_{n}\right) u_{n}+t_{n}\left[u_{n}-g_{1}\left(u_{n}\right)+m_{1}\left(u_{n}\right)+P_{K_{1}}\left(g_{1}\left(u_{n}\right)-\rho F\left(u_{n}, v_{n}\right)+m_{1}\left(u_{n}\right)\right)\right]+t_{n} e_{n}\right\}-x^{*}\right\| \\
& \leq \\
& \quad\left\|\left(1-t_{n}\right) u_{n}+t_{n}\left[u_{n}-g_{1}\left(u_{n}\right)+m_{1}\left(u_{n}\right)+P_{K_{1}}\left(g_{1}\left(u_{n}\right)-\rho F\left(u_{n}, v_{n}\right)+m_{1}\left(u_{n}\right)\right)\right]+t_{n} e_{n}-x^{*}\right\|+\epsilon_{n}^{1} .
\end{aligned}
$$


As the proof of inequality (4.11), we have

$$
\begin{aligned}
\|\{(1- & \left.\left.t_{n}\right) u_{n}+t_{n}\left[u_{n}-g_{1}\left(u_{n}\right)+m_{1}\left(u_{n}\right)+P_{K_{1}}\left(g_{1}\left(u_{n}\right)-\rho F\left(u_{n}, v_{n}\right)+m_{1}\left(u_{n}\right)\right)\right]+t_{n} e_{n}\right\}-x^{*} \| \\
\leq & \left(2 t_{n} \sqrt{1+\zeta_{1}^{2}-2 \eta_{1}}+2 t_{n} \gamma_{1}+t_{n} \sqrt{1+\rho^{2} l_{1}^{2}+2 \rho a l_{1}^{2}-2 \rho b}+1-t_{n}\right)\left\|u_{n}-x^{*}\right\| \\
& +t_{n} \rho l_{2}\left\|v_{n}-y^{*}\right\|+t_{n} b_{n}+t_{n}\left\|e_{n}\right\|,
\end{aligned}
$$

where $b_{n}$ is defined by (4.12). From (4.21) and (4.22), we have

$$
\begin{aligned}
\left\|u_{n+1}-x^{*}\right\| \leq & \left(2 t_{n} \sqrt{1+\zeta_{1}^{2}-2 \eta_{1}}+2 t_{n} \gamma_{1}+t_{n} \sqrt{1+\rho^{2} l_{1}^{2}+2 \rho a l_{1}^{2}-2 \rho b}+1-t_{n}\right)\left\|u_{n}-x^{*}\right\| \\
& +t_{n} \rho l_{2}\left\|v_{n}-y^{*}\right\|+t_{n} b_{n}+t_{n}\left\|e_{n}\right\|+\epsilon_{n}^{1} .
\end{aligned}
$$

Similarly, we have

$$
\begin{aligned}
\left\|v_{n+1}-y^{*}\right\| \leq & \left(2 t_{n} \sqrt{1+\zeta_{2}^{2}-2 \eta_{2}}+2 t_{n} \gamma_{2}+t_{n} \sqrt{1+\rho^{2} n_{2}^{2}+2 \rho c n_{2}^{2}-2 \rho d}+1-t_{n}\right)\left\|v_{n}-y^{*}\right\| \\
& +t_{n} \rho n_{1}\left\|u_{n}-x^{*}\right\|+t_{n} c_{n}+t_{n}\left\|j_{n}\right\|+\epsilon_{n}^{2}
\end{aligned}
$$

where $c_{n}$ is defined by (4.14). As the proof of inequality (4.17), and since $0<t \leq t_{n},(4.23)$ and (4.24) yield

$$
\begin{aligned}
\| u_{n+1} & -x^{*}\|+\| v_{n+1}-y^{*} \| \\
& \leq\left(1-(1-h) t_{n}\right)\left(\left\|u_{n}-x^{*}\right\|+\left\|v_{n}-y^{*}\right\|\right)+t_{n}\left(b_{n}+c_{n}+\left\|e_{n}\right\|+\left\|j_{n}\right\|\right)+\epsilon_{n}^{1}+\epsilon_{n}^{2} \\
& \leq\left(1-(1-h) t_{n}\right)\left(\left\|u_{n}-x^{*}\right\|+\left\|v_{n}-y^{*}\right\|\right)+t_{n}\left[b_{n}+c_{n}+\left\|e_{n}\right\|+\left\|j_{n}\right\|+\left(\epsilon_{n}^{1}+\epsilon_{n}^{2}\right) / t\right] \\
& =\left(1-(1-h) t_{n}\right)\left(\left\|u_{n}-x^{*}\right\|+\left\|v_{n}-y^{*}\right\|\right)+(1-h) t_{n} \delta_{n},
\end{aligned}
$$

where

$$
\delta_{n}=\frac{b_{n}+c_{n}+\left\|e_{n}\right\|+\left\|j_{n}\right\|+\left(\epsilon_{n}^{1}+\epsilon_{n}^{2}\right) / t}{1-h} .
$$

Suppose that $\lim \epsilon_{n}^{1}+\epsilon_{n}^{2}=0$, then from $b_{n} \rightarrow 0, c_{n} \rightarrow 0,\left\|e_{n}\right\| \rightarrow 0$ and $\left\|j_{n}\right\| \rightarrow 0$, we have $\delta_{n} \rightarrow 0($ as $n \rightarrow \infty)$. Then from the fact of $t_{n} \rightarrow 0, \sum t_{n}=\infty,(4.25)$ and Lemma 2.8, we have $\lim u_{n}=x^{*}$ and $\lim v_{n}=y^{*}$. 
Conversely, suppose that $\lim u_{n}=x^{*}$ and $\lim v_{n}=y^{*}$. Then we have

$$
\begin{aligned}
\epsilon_{n}^{1}+\epsilon_{n}^{2}=\| u_{n+1}-\left\{\left(1-t_{n}\right) u_{n}+t_{n}[\right. & u_{n}-g_{1}\left(u_{n}\right)+m_{1}\left(u_{n}\right) \\
& \left.\left.+P_{K_{1}}\left(g_{1}\left(u_{n}\right)-\rho F\left(u_{n}, v_{n}\right)+m_{1}\left(u_{n}\right)\right)\right]+t_{n} e_{n}\right\} \| \\
+ & \| v_{n+1}-\left\{\left(1-t_{n}\right) v_{n}+t_{n}\left[v_{n}-g_{2}\left(v_{n}\right)+m_{2}\left(v_{n}\right)\right.\right. \\
& \left.\left.+P_{K_{2}}\left(g_{2}\left(v_{n}\right)-\rho G\left(u_{n}, v_{n}\right)+m_{2}\left(v_{n}\right)\right)\right]+t_{n} j_{n}\right\} \| \\
\leq & \left\|u_{n+1}-x^{*}\right\|+\|\left(1-t_{n}\right) u_{n}+t_{n}\left[u_{n}-g_{1}\left(u_{n}\right)+m_{1}\left(u_{n}\right)\right. \\
& \left.+P_{K_{1}}\left(g_{1}\left(u_{n}\right)-\rho F\left(u_{n}, v_{n}\right)+m_{1}\left(u_{n}\right)\right)\right] \\
& +t_{n} e_{n}-x^{*}\|+\| v_{n+1}-y^{*} \| \\
& \left.\quad+P_{K_{2}}\left(g_{2}\left(v_{n}\right)-\rho G\left(u_{n}, v_{n}\right)+m_{2}\left(v_{n}\right)\right)\right]+t_{n} j_{n}-y^{*} \| v_{n}+t_{n}\left[v_{n}-g_{2}\left(v_{n}\right)+m_{2}\left(v_{n}\right)\right. \\
\leq & \left\|u_{n+1}-x^{*}\right\|+\left\|v_{n+1}-y^{*}\right\|+t_{n} \rho l_{2}\left\|v_{n}-y^{*}\right\|+t_{n} \rho n_{1}\left\|u_{n}-x^{*}\right\| \\
& +\left(2 t_{n} \sqrt{1+\zeta_{1}^{2}-2 \eta_{1}}+2 t_{n} \gamma_{1}+t_{n} \sqrt{1+\rho^{2} l_{1}^{2}+2 \rho a l_{1}^{2}-2 \rho b}+1-t_{n}\right)\left\|u_{n}-x^{*}\right\| \\
& +\left(2 t_{n} \sqrt{1+\zeta_{2}^{2}-2 \eta_{2}}+2 t_{n} \gamma_{2}+t_{n} \sqrt{1+\rho^{2} n_{2}^{2}+2 \rho c n_{2}^{2}-2 \rho d}+1-t_{n}\right)\left\|v_{n}-y^{*}\right\| \\
& +t_{n} b_{n}+t_{n}\left\|e_{n}\right\|+t_{n} c_{n}+t_{n}\left\|j_{n}\right\|
\end{aligned}
$$

and so $\epsilon_{n}^{1}+\epsilon_{n}^{2} \rightarrow 0$ as $n \rightarrow \infty$. This completes the proof.

\section{Acknowledgments}

The authors thank the referees for their valuable suggestions. This work was supported by the National Natural Science Foundation of China and the Educational Science Foundation of Chongqing (KJ051307).

\section{References}

[1] R. P. Agarwal, Y. J. Cho, J. Li, and N. J. Huang, Stability of iterative procedures with errors approximating common fixed points for a couple of quasi-contractive mappings in $q$-uniformly smooth Banach spaces, Journal of Mathematical Analysis and Applications 272 (2002), no. 2, 435-447.

[2] R. P. Agarwal, N. J. Huang, and Y. J. Cho, Generalized nonlinear mixed implicit quasi-variational inclusions with set-valued mappings, Journal of Inequalities and Applications 7 (2002), no. 6, $807-828$.

[3] R. P. Agarwal, N. J. Huang, and M. Y. Tan, Sensitivity analysis for a new system of generalized nonlinear mixed quasi-variational inclusions, Applied Mathematics Letters 17 (2004), no. 3, 345352. 
[4] C. Baiocchi and A. Capelo, Variational and Quasivariational Inequalities, Application to Free Boundary Problems, A Wiley-Interscience Publication, John Wiley \& Sons, New York, 1984.

[5] J. Y. Chen, N. C. Wong, and J. C. Yao, Algorithm for generalized co-complementarity problems in Banach spaces, Computers \& Mathematics with Applications 43 (2002), no. 1-2, 49-54.

[6] Y. J. Cho, Y. P. Fang, N. J. Huang, and H. J. Hwang, Algorithms for systems of nonlinear variational inequalities, Journal of the Korean Mathematical Society 41 (2004), no. 3, 489-499.

[7] Y. P. Fang and N. J. Huang, Variational-like inequalities with generalized monotone mappings in Banach spaces, Journal of Optimization Theory and Applications 118 (2003), no. 2, 327-338.

[8] __ Existence results for systems of strongly implicit vector variationa inequalities, Acta Mathematica Hungarica 103 (2004), no. 4, 265-277.

[9] N. J. Huang, On the generalized implicit quasivariational inequalities, Journal of Mathematical Analysis and Applications 216 (1997), no. 1, 197-210.

[10] N. J. Huang, M. R. Bai, Y. J. Cho, and S. M. Kang, Generalized nonlinear mixed quasi-variational inequalities, Computers \& Mathematics with Applications 40 (2000), no. 2-3, 205-215.

[11] C. R. Jou and J. C. Yao, Algorithm for generalized multivalued variational inequalities in Hilbert spaces, Computers \& Mathematics with Applications 25 (1993), no. 9, 7-16.

[12] G. Kassay and J. Kolumbán, System of multi-valued variational inequalities, Publicationes Mathematicae Debrecen 56 (2000), no. 1-2, 185-195.

[13] G. Kassay, J. Kolumbán, and Z. Páles, Factorization of Minty and Stampacchia variational inequality systems, European Journal of Operational Research 143 (2002), no. 2, 377-389.

[14] J. K. Kim and D. S. Kim, A new system of generalized nonlinear mixed variational inequalities in Hilbert spaces, Journal of Convex Analysis 11 (2004), no. 1, 235-243.

[15] R. U. Verma, Projection methods, algorithms, and a new system of nonlinear variational inequalities, Computers \& Mathematics with Applications 41 (2001), no. 7-8, 1025-1031.

[16] _ Generalized system for relaxed coercive variational inequalities and projection methods, Journal of Optimization Theory and Applications 121 (2004), 203-210.

[17] X. Weng, Fixed point iteration for local strictly pseudo-contractive mapping, Proceedings of the American Mathematical Society 113 (1991), no. 3, 727-731.

[18] S. Z. Zhou, Perturbation for elliptic variational inequalities, Science in China (Scientia Sinica). Series A 34 (1991), no. 6, 650-659.

Ke Ding: Department of Mathematics, Sichuan University, Chengdu, Sichuan 610064, China E-mail address: keding@yahoo.com

Wen-Yong Yan: Department of Mathematics, Sichuan University, Chengdu, Sichuan 610064, China E-mail address: wenyongy@yahoo.com

Nan-Jing Huang: Department of Mathematics, Sichuan University, Chengdu, Sichuan 610064, China E-mail addresses: nanjinghuang@126.com; nanjinghuang@hotmail.com 